\title{
Influence(s) du contexte géographique en sciences physiques sur la construction d'un concept scientifique au lycée
}

\author{
Pierre FLEURY \\ Nouvelle Université de Bordeaux
}

\section{Résumé}

Nous interrogeons, en sciences physiques, pour un élève de seconde générale, le paramètre « contexte géographique»" comme pouvant influencer la manière d'atteindre un niveau donné de construction d'un concept scientifique. Nous nous appuyons sur l'apport de Vergnaud (1985) pour déterminer les corrélations existantes entre Concept et invariant(s) associé(s) et ainsi définir les niveaux de constructions du concept atteints par les élèves. L'étude est menée à partir d'une analyse de contenu sur les représentations produites par les sujets au cours de l'apprentissage, lesquelles sont passées par le filtre de la catégorisation proposée par Sallaberry (1996). Le paramètre contexte testé ici est de nature géographique (les deux échantillons étant d'origine métropolitaine et martiniquaise). L'étude montre que si des différences sont observables dans les représentations construites (le processus), le paramètre « contexte géographique» ne semble pas avoir d'influence sur l'intimité de la liaison invariant-concept (le produit) pour le concept d'élément chimique.

\section{Mots-clés}

Contexte géographique, élément chimique, invariant, représentations, sciences physiques.

\begin{abstract}
We examine what is, in the area of physical science, for a year 11 student, the possible influence of the "geographical context» in the reaching of a given level in the development of a scientific concept. We rely on the work of Vergnaud (1985) to determine the existing links between Concept and associated invariants and so define the levels reached by the student in the construction of the concept. The study starts from an anlysis of the content of the representations reached by the students in the course of their learning process, which follows the categories proposed by Salaberry (1996). The context parameter tested here is geographical in nature (the samples originating from France and Martinique). The study shows that if differences can be observed in the constructed representation (the process), the geographical context does not seem to have any influence on the strenght of the link constant-concept ( the process) for the concept of chemical element.
\end{abstract}

\section{Keywords}

Geographical context, chemical element, invariant, representations, physical sciences

\footnotetext{
${ }^{1}$ À travers ses dimensions culturelles et scolaires.
} 


\section{Introduction}

Un des objectifs de l'enseignement des sciences physiques est la délicate construction des objets complexes que sont les concepts scientifiques par les élèves. Si la démarche par investigation aujourd'hui promue par l'institution est une réponse possible, nous pensons qu'aborder la question des concepts est liée à celle de ses invariants associés (Vergnaud, 1985).

Nous formulons l'hypothèse que construire l'invariant ou les invariants liés à un concept scientifique est de nature à lui donner une meilleure lisibilité. Ce travail de recherche propose d'observer en quoi l'influence du paramètre «contexte géographique» peut jouer sur la qualité de la liaison invariant-concept, dans la construction du concept d'élément chimique au lycée en classe de seconde générale.

\section{La construction d'un concept}

\subsection{Définition et caractérisation du concept d'élément chimique}

Interroger le cadrage théorique de l'élément chimique est une nécessité. En effet, la lecture des nouveaux programmes de seconde générale ${ }^{2}$ d'une part, ainsi que les propositions des manuels scolaires destinés aux élèves révèlent une réelle difficulté à aborder ce concept. Dès la programmation de ce concept au collège (en $5^{\circ}$ ) à la fin des années 1970 , sont apparues des confusions importantes entre ce qui relève de la définition du concept et ce qui est du domaine de sa caractérisation. Martinand (1986) nous éclaire à ce sujet en précisant cette distinction. Il pose comme préalable qu'un élément chimique se définit à travers les transactions électroniques lors des transformations chimiques. Il ne saurait donc être question d'avoir recours à la structure de la matière (nombre de protons) pour définir le concept. Pour autant, s'intéresser au noyau informe sur la caractérisation du concept et lui donne une autre lisibilité.

Cela étant posé, force est de constater qu'il persiste au mieux une ambigüité entre définir et caractériser le concept d'élément chimique. Ce piège peut être entre autres révélé par l'utilisation à mauvais escient qui est faite des deux verbes précédents (caractériser à la place de définir). Les approches «chimiques» et «structurales» sont complémentaires voire indispensables pour prétendre «tenir » le concept d'élément chimique. Toutefois la première relève de la définition du concept quand la seconde aborde sa caractérisation. Alors que le concept d'élément chimique «appartient» aux domaines des électrons, il se trouve très souvent « propulsé » vers celui des protons et de la physique nucléaire !

Les divers travaux en formation des maîtres (Sallaberry, 2000) avec des enseignants de lycées et collèges témoignent de ce transit intellectuel qui s'observe de fait dans les cahiers des élèves. Bien que ce concept ne trouve pas de réel rayonnement dans la communauté scientifique $^{3}$ aujourd'hui, il trouve de nouveau toute sa place dans les programmes nouvellement réformés de la première année du lycée général. Si les obstacles (Laugier et

\footnotetext{
${ }^{2} \mathrm{BO}$ spécial $\mathrm{n}^{\circ} 4$ du 29 avril 2010 qui révèle que, si la notion de caractérisation est correctement abordée à partir de la structure du noyau (nombre de protons), on observe en revanche une ambiguïté sur l'utilisation du terme «élément» qui est parfois utilisé pour désigner le concept d'élément chimique et parfois pour désigner un élément chimique particulier.

3 Propos tenus par Martinand durant un atelier sur la formation, au congrès de l'AFIRSE (Association Francophone Internationale de Recherche Scientifique en Éducation - Paris, siège de l'UNESCO, Juin 2011).
} 
Dumon, 2003) liés à son apprentissage sont nombreux, il reste digne d'intérêt de s'intéresser aux conditions didactiques susceptibles de le rendre plus lisible : confronter ce concept à son invariant par conservation-transformation. Nous retiendrons donc, s'agissant de la définition et de la caractérisation de ce concept les formulations suivantes proposées par Martinand (1986) :

- Définition de l'élément chimique : «L'élément chimique est ce qui se conserve lors d'une transformation chimique »

- Caractérisation de l'élément chimique : «Ensemble des espèces chimiques ayant le même numéro atomique $(\mathrm{Z})$ ou nombre de protons »

\subsection{Les deux approches de VERGNAUD}

Vergnaud (1985) engage une réflexion sur la lisibilité des concepts. Il propose pour le concept une structure de type $(\mathbf{S}, \mathbf{I}, \mathbf{S})$ :

- $\quad \mathbf{S}$ : ensemble des situations qui donnent du sens au concept ;

- I : ensemble des invariants opératoires qui sont sous-jacents au traitement de ces situations par le sujet;

- S : ensemble des signifiants (ou symbolisations) qui permettent de représenter les invariants, les situations, les procédures du traitement (Vergnaud, $1985: 247-248$ ).

Ses travaux en mathématiques le conduisent $\left(1991^{4}\right)$ à définir une théorie dite « des champs conceptuels ».Selon lui, la définition d'un concept ne se suffisant pas à elle-même, il faut aborder un concept scientifique en ayant le souci de prévoir un ensemble de situations qui saura lui donner du sens (un ensemble d'opérations à concevoir sous forme expérimentale en Sciences physiques). Lors de ces opérations, il faudra prendre le soin qu'un ou plusieurs invariants (ce qui implique de les avoir préalablement identifiés) puisse(nt) émerger, ce qui facilitera l'appropriation du concept. Enfin, un temps significatif individuel devra être destiné à l'élaboration, l'expression, des symboles et des procédures qui accompagneront la construction du concept.

\subsection{La question de recherche}

Un premier travail sur la liaison invariant-concept abordant le concept d'élément chimique a été présenté lors du congrès international de l'AFIRSE $^{5}$; les premiers résultats semblent montrer qu'il existe un lien étroit entre les niveaux de construction, du concept d'élément chimique et de son invariant par conservation-transformation. Nous tentons donc d'observer dans quelle mesure ce paramètre pourrait influencer le type de représentations produites (processus) d'une part, et l'intimité de la liaison invariant-concept (produit) laquelle détermine de fait le niveau de construction du concept atteint d'autre part.

\footnotetext{
${ }^{4}$ Vergnaud (1991) pp.133-170.

${ }^{5}$ Communication proposée par Fleury P. Invariant(s) et Concept en situation de formation (le cas du concept d'élément chimique) laquelle met en avant l'intimité de la liaison invariant-concept et son intérêt didactique.
} 


\section{Le protocole ${ }^{6}$ expérimental}

On trouve dans les laboratoires de lycées de nombreux protocoles basés sur un système de boucle réactionnelle laquelle peut être résumée (pour l'élément chimique cuivre) sous la forme de l'organigramme ci-dessous (cf. Figure 1).

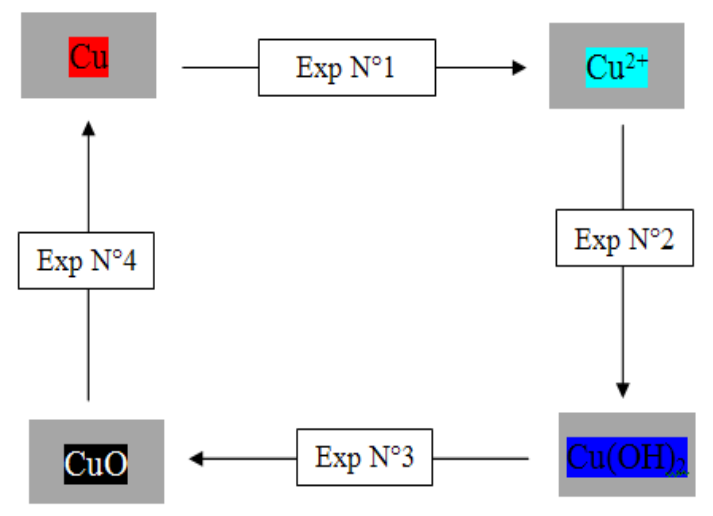

Figure 1 : Boucle réactionnelle

Le protocole expérimental est organisé autour d'une série de quatre expériences qui ont été réalisées en binôme. Les transformations chimiques mises en jeu sont singulières dans la mesure où le ou l'un des produits formés «devient » l'un des réactifs de la transformation suivante.

Ainsi, le protocole est élaboré dans une logique de faire émerger l'invariant de par la nature cyclique de l'ensemble. L'idée de conservation est donc implicite dans cette mise en scène opératoire. L'expérience met en jeu quatre espèces chimiques différentes contenant l'élément cuivre dans l'ordre chronologique suivant: $(\mathrm{Cu}),\left(\mathrm{Cu}^{2+}\right),\left(\mathrm{Cu}(\mathrm{OH})_{2}\right)$ et $(\mathrm{CuO})$.

\section{Le recueil des corpus ${ }^{7}$}

L'expérience a été réalisée en seconde générale aux lycées Arnaut Daniel ${ }^{8}$ et Bellevue ${ }^{9}$ pendant une séance de travaux pratiques sur deux groupes de quatorze élèves (vingt-huit au total) en novembre 2010 et septembre 2011. Le concept d'élément chimique est abordé en débutant par le même protocole expérimental ci-dessus. Les élèves ont réalisé les expériences à partir d'un même document qui servait de protocole général. À titre individuel, ils ont rempli un même document par expérience, partagé en trois zones, leur demandant de schématiser, de rendre compte de leurs observations, et de donner des interprétations susceptibles d'éclairer l'ensemble.

En fin de séance, chaque élève a été invité, à répondre à la question : "Que pensez-vous de cette séries d'expériences? »(Bilan personnel sur un même document à part). L'analyse du corpus porte donc sur l'ensemble des productions individuelles construites pendant la séance, ce qui représente quatorze dossiers individuels par classe. Un dossier est constitué de 5 feuilles A4 (une par expérience et une synthèse).

\footnotetext{
${ }^{6}$ Voir annexe 1.

${ }^{7}$ Voir annexes 2 et 3 pour les supports distribués aux élèves sur lesquels ils ont élaboré leurs représentations.

${ }^{8}$ Etablissement situé en zone rurale sur la commune de Ribérac (Dordogne-24).

${ }^{9}$ Etablissement situé en zone insulaire sur la commune de Fort de France (Martinique-972).
} 
Tout au long du protocole les élèves avaient en leur possession un tableau ${ }^{10}$ de données sur les différentes espèces chimiques mise en jeu.

\section{La méthode d'analyse}

\subsection{La catégorisation des représentations}

Nous retiendrons pour cette étude, la catégorisation de Sallaberry (2004), qui présente quatre classements distincts dont nous utiliserons les exploitables (R1/R2/R1-R2), les représentations qu'il qualifie d'inconscientes ne nous étant pas accessibles.

- S'agissant des représentations codées R1 : [Les R1 ...ont des bords flous et installent, entre elles, un fonctionnement caractérisé par l'imprécision. Le propre d'une image, qu'elle soit picturale ou décrite à l'aide de mots, est justement de toujours faire penser à une autre $]^{14}$. Ces représentations que l'on pourrait définir comment étant «descriptives» ont pour vocation au cours d'un protocole expérimental d'acter les différents observables et de se poser comme un préalable aux R2.

Exemple tiré du corpus :

"Il y a des petits points rouges sur le tube à essais »

- S'agissant des représentations codées $\mathrm{R} 2$ : [Les $R 2$ ont vocation à utiliser ou à devenir des concepts]. Ces représentations ont des visées «interprétatives »et témoignent d'un autre niveau de la pensée.

Exemple tiré du corpus :

"Je crois que les parois du tube sont recouvertes d'un solide »

- S'agissant des coordinations R1- R2 (R3) : elles mettent en lien des observables ou relèvent de passerelles entre un vécu immédiat et des « imaginaires » en construction (on pourra par ailleurs noter qu'il existe de nombreuses représentations « composites » qui combinent les propriétés des R1 et des R2). Ces représentations sont toujours une conjecture : on peut les concevoir comme des représentations pour lesquelles le sujet qui les produit franchit un palier important sur le plan conceptuel. On pourrait avancer que ces représentations témoignent d'un niveau intéressant de la construction d'un concept.

Exemple tiré du corpus :

"On voit des dépôts rouges donc cela pourrait être du métal cuivre reformé "

\subsection{Le repérage d'indices}

Si les représentations qui relèvent des strictes descriptions (codées R1) ne posent pas de problème s'agissant de leur repérage, les représentations à visées interprétatives peuvent être identifiées à partir d'indices lexicaux. Ainsi, les conjonctions de coordinations, certains adverbes, l'emploi du subjonctif ou du conditionnel, mais également toutes tentatives d'explications, d'hypothèses sont autant de symptômes qui témoignent d'un engagement cognitif relevant des représentations rationnelles (codées R2 et R1-R2).

\footnotetext{
${ }^{10}$ Voir annexe 4.
} 
Nous avons analysé la présence de ces indices (liste ci-dessous) de manière systématique par analyse de contenu dans chaque phrase construite :

- la présence d'un connecteur logique : conjonction de coordination (codé $\mathbf{C c}$ ) ou de toute autre nature (conjonction de subordination, adverbe...) (codé Ac) ;

- l'utilisation de temps spécifiques : conditionnel présent (codé $\mathbf{C l}$ ), subjonctif présent (codé $\mathbf{S f}$ ), participe présent $(\mathbf{P p})$;

- la présence d'une figure de style (codé Fs);

- la présence d'une expression qui débouche sur une tentative d'explication ou qui relève d'un raisonnement (codé Te-R);

- la trace, du démarrage d'une hypothèse (codé : Hy) ou d'indicateurs Hypothèse.

Nous donnons quelques exemples tirés du corpus qui illustrent les différents indices lexicaux retenus pour cette analyse.

- $\quad$ En effet (Te-R), tout comme l'huile dans l'eau (Fs), il est probable (Hy) que l'ion cuivre II ne puisse pas (Sf) se diluer avec la solution concentrée...].

- [..Il n'y a donc (Cc) plus d'oxygène dans l'oxyde de cuivre. Or $(C c)$, l'oxyde de cuivre se base avec le cuivre et l'oxygène. Ainsi (Ac), le cuivre réapparaît sans Oxygène].

- [Normalement (Ac), le filtrat obtenu aurait dîu (Cl) être transparent, je pense (Hy)

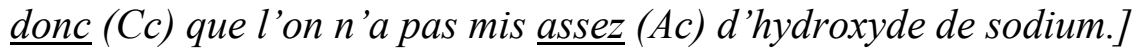

- [L'oxygène ayant disparu (Pp,) l'oxyde de cuivre à forte température devient ou (Hy) redevient métal cuivre car $(C c)$ ce qui est obtenu dans le tube à essai a une couleur orangée]

\subsection{Les propositions ${ }^{11}$ de catégorisations de l'invariant et du concept}

La construction de l'invariant s'apprécie à partir de sa présence (ici les espèces chimiques contenant l'élément cuivre) au cours du protocole expérimental et par conséquent nous recherchons des indices d'une continuité de présence. La construction du concept d'élément s'apprécie entre autres à partir de l'idée de sa conservation tout au long du protocole. Ainsi la seule idée de continuité de présence ne peut, à elle seule, préjuger de l'idée de conservation lors d'une transformation.

\begin{tabular}{|l|l|}
\hline \multicolumn{2}{|c|}{ Niveaux $\left(\mathbf{I}_{\mathbf{x}}\right)$ de construction de l'invariant } \\
\hline $\mathbf{I}_{\mathbf{1}}$ & La continuité de la présence de « l'espèce cuivre » est absente. \\
\hline $\mathbf{I}_{\mathbf{2}}$ & $\begin{array}{l}\text { La continuité de la présence de « l'espèce cuivre » est abordée. } \\
\text { Ex Idée de retrouvaille, de reconstitution - Description avec les espèces chimiques }\end{array}$ \\
\hline $\mathbf{I}_{3}$ & $\begin{array}{l}\text { La continuité de la présence de « l'espèce cuivre » est implicite. } \\
\text { Ex Non disparition - Traces permanentes - Représentations coordonnant les espèces } \\
\text { chimiques entre elles }\end{array}$ \\
\hline $\mathbf{I}_{\mathbf{4}}$ & La continuité de la présence de « l'espèce cuivre » est explicitée. \\
\hline
\end{tabular}

Tableau 1 : Catégorisations retenues pour l'invariant

\footnotetext{
11 Ces deux propositions de catégorisation sont construites à partir des spécificités de l'invariant par conservation-transformation d'une part et du concept d'élément chimique d'autre part.
} 
Nous proposons d'identifier trois indices essentiels à partir desquels nous pouvons proposer de catégoriser le concept :

1- Cycle/ Boucle / Description cyclique / Écritures symboliques en boucle ou cycle

2- Transformations / Changement de formes

3- Cuivre sous différentes formes (états)

Nous proposons de codifier les catégorisations du concept, au regard des présences au sein du corpus de ces trois « familles d'indices ».

\begin{tabular}{|l|l|}
\hline \multicolumn{2}{|c|}{ Niveaux (Cy) de construction du concept } \\
\hline C1 & Aucun des trois indices n'est présent \\
\hline C2 & Présence d'un des trois indices \\
\hline C3 & Présence de deux des trois indices \\
\hline C4 & Présence des trois indices mais absence d'explicitation et de généralisation \\
\hline C5 $^{(*)}$ & $\begin{array}{l}\text { Présence des trois indices avec explicitation - la conservation peut être explicitée. } \\
(\text { et si généralisation }(*)) \text { [ ça vaut le coup de parler d'élément »] }\end{array}$ \\
\hline
\end{tabular}

Tableau 2 : Catégorisations retenues pour le concept

Muni de ces deux catégorisations, nous pouvons établir les liaisons invariant-concept atteintes par chaque élève à l'issue du protocole expérimental, lesquelles sont établies à partir de l'analyse des dossiers.

\section{Les résultats}

6.1. Niveau $\left(C_{y}\right)$ de construction atteint du concept, au regard de celui $\left(I_{x}\right)$ de l'invariant : liaisons $\left(\mathrm{I}_{\mathrm{x}}-\mathrm{C}_{\mathrm{y}}\right)$ invariant-concept

Les résultats des deux échantillons sont regroupés dans un même tableau (ci-dessous). Les occurrences du groupe métropolitain («24») sont notées en italique, celles du groupe martiniquais («972») sont données entre parenthèse. On observe ainsi que les deux groupes ont construit chacun neuf liaisons invariant-concept différentes dont cinq sont communes aux deux échantillons $\left(\mathbf{I}_{2}-\mathbf{C}_{2}\right) /\left(\mathbf{I}_{2}-\mathbf{C}_{3}\right) /\left(\mathbf{I}_{3}-\mathbf{C}_{2}\right) /\left(\mathbf{I}_{3}-\mathbf{C}_{3}\right)$ et $\left(\mathbf{I}_{4}-\mathbf{C}_{3}\right)$.

\begin{tabular}{|c|c|c|c|c|c|c|c|}
\hline & $\mathrm{C}_{1}$ & $\mathrm{C}_{2}$ & $\mathrm{C}_{3}$ & $\mathrm{C}_{4}$ & $\mathrm{C}_{5}$ & $\mathrm{C}_{5}{ }^{*}$ & TOTAL \\
\hline $\mathbf{I}_{1}$ & 1 & & & & & & 1 \\
\hline$I_{2}$ & 2 & 2(3) & l(2) & (1) & & & 11 \\
\hline$\overline{I_{3}}$ & & l(1) & $1(2)$ & & & & 5 \\
\hline$I_{4}$ & (1) & (1) & $1(2)$ & (1) & 2 & 3 & 11 \\
\hline
\end{tabular}

Tableau 3 : Les résultats des liaisons invariants-concepts $\left(I_{x}-C_{y}\right)$ pour les deux échantillons

- Seul un élève n'a réussi à aborder ni l'invariant ni le concept $\left[\mathbf{I}_{\mathbf{1}}-\mathbf{C}_{\mathbf{1}}\right]$.

- Deux élèves sont en train de construire l'invariant mais pas le concept $\left[\mathbf{I}_{2}-\mathbf{C}_{1}\right]$.

- Un élève a construit l'invariant sans investir le concept $\left[\mathbf{I}_{\mathbf{4}}-\mathbf{C}_{\mathbf{1}}\right]$. 
- Quatorze élèves sont en train de construire l'invariant et le concept $\left(\left[\mathbf{I}_{2}-\mathbf{C}_{2}\right]-\left[\mathbf{I}_{2}-\mathbf{C}_{3}\right]-\right.$ $\left.\left[\mathbf{I}_{2}-\mathbf{C}_{4}\right]-\left[\mathbf{I}_{3}-\mathbf{C}_{2}\right]-\left[\mathbf{I}_{3}-\mathbf{C}_{3}\right]\right)$ : ces élèves forment le groupe majoritaire ce qui nous semble assez logique compte tenu des circonstances d'apprentissages.

- Cinq élèves ont construit l'invariant et sont en train de construire le concept $\left[\mathbf{I}_{\mathbf{4}}-\mathbf{C}_{\mathbf{2}}\right]-$ $\left[\mathbf{I}_{4}-\mathbf{C}_{3}\right]-\left[\mathbf{I}_{\mathbf{4}}-\mathbf{C}_{\mathbf{4}}\right]$.

- Cinq élèves ont construit l'invariant et le concept $\left[\mathbf{I}_{4}-\mathbf{C}_{5}\right]-\left[\mathbf{I}_{4}-\mathbf{C}_{5}{ }^{*}\right]$.

Nous actons l'absence des liaisons $\left[\mathbf{I}_{\mathbf{3}}-\mathbf{C}_{\mathbf{4}}\right.$ et $\mathbf{a u}$-delà $]$ qui pourraient illustrer l'importance de l'invariant dans la construction du concept. Nous observons par ailleurs l'absence des liaisons [ $\mathbf{I}_{1}-\mathbf{C}_{\mathbf{2}}$ et au-delà $]$ mais aussi $\left[\mathbf{I}_{2}-\mathbf{C}_{5}\right.$ et au-delà $]$ ce qui nous paraît plus cohérent dans une logique d'intimité.

On pourra également remarquer au second ordre, que deux liaisons particulières sont obtenues pour l'échantillon «972». La liaison (4-1) pour laquelle l'élève a identifié l'invariant sans pour autant commencer à construire le concept (l'élève a confondu cuivre métal et cuivre "élément »); la liaison (2-4) où l'invariant est au début de sa construction alors que le concept est sur le point d'être atteint (cet élève a eu beaucoup de difficulté à identifier les différentes espèces mises en jeu). En outre l'échantillon (« $24 »)$ présente deux liaisons exclusives pour lesquelles ces élèves sont parvenus à identifier l'invariant et le concept (4-5) voire généraliser l'invariant (4-5*) indiquant alors que l'idée de conservation n'est pas une propriété unique de l'élément chimique cuivre.

En outre, nous ne trouvons dans aucun des deux échantillons « 24 » et « 972 », de liaison qui indiquerait qu'il est envisageable de tenir parfaitement le concept $\left(\mathrm{C}_{5}\right)$ sans avoir tenu au préalable l'invariant $\left(\mathrm{I}_{4}\right)$. Par ailleurs, le fait de ne pas construire l'invariant $\left(\mathrm{I}_{1}\right)$ apparaît comme un obstacle pour construire le concept. Enfin, dès l'amorce $\left(\mathrm{I}_{2}\right)$ de la construction de l'invariant, la construction du concept est très majoritairement enclenchée $\left(\mathrm{C}_{2}\right.$ à $\left.\mathrm{C}_{4}\right)$. Nous avons avec ces résultats des indications particulièrement intéressantes s'agissant de l'intimité entre le concept étudié et son invariant, qui semblent bien s'affranchir du paramètre contexte.

\subsection{Répartition des représentations au cours des différentes expériences}

Nous recherchons désormais si des caractéristiques liées au contexte se dégagent, dans les représentations élaborées en cours d'apprentissage par les élèves.

Un état des lieux, reprenant les étapes du dossier, expérience par expérience puis bilan, avec enfin des moyennes par élève et par catégories de représentation, est donné dans le tableau 4.

- Pour l'échantillon «972», lors de l'expérience 1 (laquelle permet de transformer le métal cuivre en ions cuivre II), les élèves ont construit $3 \mathrm{R} 1$ (42 au total /14 élèves), et environ 2R2 et 2R1-R2, soit au total environ 7 représentations.

- Pour l'échantillon « 24 », lors de l'expérience 4, 2R1, environ 5R2 et 2R1-R2 ont été construites. 


\begin{tabular}{|c|c|c|c|c|c|c|c|}
\hline \begin{tabular}{|c|} 
Bilan \\
échantillon \\
$« 972 »$ \\
\end{tabular} & $R 1$ & $R 2$ & $R 1-R 2$ & \begin{tabular}{|c|} 
Bilan \\
échantillon \\
$\ll 24 »$ \\
\end{tabular} & $R 1$ & $R 2$ & $R 1-R 2$ \\
\hline Expérience 1 & 42 & 29 & 20 & Expérience 1 & 46 & 39 & 16 \\
\hline Expérience 2 & 36 & 36 & 27 & Expérience 2 & 38 & 37 & 20 \\
\hline Expérience 3 & 19 & 15 & 11 & Expérience 3 & 45 & 53 & 15 \\
\hline Expérience 4 & 18 & 36 & 27 & Expérience 4 & 25 & 74 & 30 \\
\hline Les bilans & 3 & 40 & 26 & Les bilans & 0 & 86 & 30 \\
\hline $\begin{array}{c}\text { Total } \\
\text { (Moy/él) }\end{array}$ & $118(8-9)$ & $156(11-12)$ & $91(6-7)$ & $\begin{array}{c}\text { Total } \\
\text { (Moy/él) }\end{array}$ & 154(11) & $250(17-18)$ & $111(8)$ \\
\hline
\end{tabular}

\section{Tableau 4 : Bilan des représentations produites par les deux échantillons « 972 » et « 24 » selon les critères de la catégorisation R1 - R2 - R1/R2}

L'échantillon « $24 »$ a produit un nombre plus important de représentations sur les trois catégories avec un écart significatif $(+60 \%)$ en ce qui concerne les $\mathrm{R} 2,(+30 \%)$ pour les $\mathrm{R} 1$ et $(+20 \%)$ pour les R1-R2.

L'analyse des R1 fait apparaître que l'échantillon « 972 » a recours aux couleurs de manière très prégnante (environ 20 occurrences colorées par élève contre 12 seulement pour les élèves « $24 »)$. La gamme des couleurs est très étendue et plus précise jouant ainsi sur des teintes, sur des doubles adjectifs (bleu-ciel, turquoise, foncé, clair).

Exemple tiré du corpus :

"Après avoir versé l'acide nitrique sur la tournure de cuivre, le liquide s'est mis en ébullition et de la fumée marron s'évaporait du tube pendant que celui-ci prenait la couleur marron. Son liquide était vert. A la fin de l'expérience, on distinguait deux caractéristiques, le tube était marron et le liquide bleu foncé ».

On peut avancer que les descriptions sont plus fidèles et plus précises de manière générale pour ces élèves. Par ailleurs, quelques R1 spécifiques ne se retrouvent pas d'un échantillon à un autre. Pour l'échantillon « $24 »$ ( «bouillie bordelaise » - "vert comme un étang »); pour l'échantillon «972» («comme de la mousse» - "une sorte de bouchon dans l'écume» " comme une méduse »); ce qui peut être le révélateur d'un contexte géographique différent. Au contraire des R1 comme ("masse gélatineuse » - "résidus noir » - " nuage bleu ») sont données par les deux groupes.

On peut enfin noter que le bilan rédigé en fin de séance reste le siège des interprétations (R2), les R1 n'étant plus présentes (ou de manière très marginale avec 3R1 pour le groupe « 972 ») pour les deux groupes :

- L'analyse des R2 fait émerger que le groupe « $24 »$ a passé plus de temps à « expliquer» en particulier sur les deux dernières expériences ainsi que lors du bilan. Les indicateurs tels que les adverbes, conjonctions de coordinations, tentatives d'explications, voire hypothèses sont beaucoup plus utilisés $(+50 \%$ en moyenne par élève).

- L'analyse des R1-R2 pour le groupe « $972 »$, témoigne d'un souci des élèves d'être concentrés sur les aspects réactionnels comme les changements d'état plutôt que sur les identifications des composés chimiques mis en jeu.

Ainsi, pour l'échantillon « 972 », seul un élève a identifié de manière explicite la présence de l'ion cuivre ; mais aucun n'a reconnu les présences de l'hydroxyde de cuivre et de l'oxyde de cuivre. En revanche pour le groupe « 24 » seuls deux élèves ne sont pas parvenus à identifier 
ces deux dernières espèces, ce qui est un résultat diamétralement opposé. S'agissant du métal cuivre obtenu en fin de réaction les deux groupes font jeu égal sur l'aptitude à le « démasquer».

Ces identifications des différentes formes de l'élément cuivre pouvaient être obtenues par associations déductives, en croisant les observations et les données du tableau distribué aux élèves.

En résumé, l'échantillon « 972 », avant d'écrire le bilan final, a plus précisément décrit (R1), mais s'est moins attaché à interpréter (R2) et ne paraît pas avoir su identifier la majorité des espèces contenant l'élément cuivre. On pouvait penser à ce stade que le groupe « 24 » avait contrairement au groupe «972», les cartes en main, pour mieux construire l'invariant, la construction du concept pouvant en être affectée. Les résultats obtenus des niveaux de construction du concept in fine n'ont pas permis de valider cette hypothèse. En effet parmi les liaisons exclusives du groupe « $24 »$, certaines sont très abouties $\left[\mathbf{I}_{\mathbf{4}}-\mathbf{C}_{\mathbf{5}}\right]-\left[\mathbf{I}_{\mathbf{4}}-\mathbf{C}_{\mathbf{5}}{ }^{*}\right]$, quand d'autres le sont beaucoup moins $\left[\mathbf{I}_{1}-\mathbf{C}_{\mathbf{1}}\right]-\left[\mathbf{I}_{2}-\mathbf{C}_{\mathbf{1}}\right]$.

\section{Conclusion}

Ces résultats attirent notre attention plus particulièrement sur les trois points suivants :

- Le paramètre «contexte géographique » ne semble pas avoir d'influence sur l'intimité de la liaison invariant-concept pour l'exemple de la construction du concept d'élément chimique par des élèves de seconde générale.

- Le paramètre «contexte géographique» en revanche semble influencer plus sensiblement les représentations descriptives (R1). On peut noter :

○ un meilleur niveau de précision pour le groupe « 972 » (en particulier dans le choix des couleurs et des teintes)

○ des expressions langagières qui identifient un environnement local spécifique (marin / rural).

- Une tendance semble se dégager qui est celle de l'importance des représentations R1. En effet bien qu'ayant moins eu recours aux représentations rationnelles (R2 et R1R2), les élèves du groupe « $972 »$ construisent le concept de manière comparable au groupe « $24 »$.

Ainsi les R1 sembleraient jouer un rôle important dans la construction d'un concept. Ceci va dans le sens de l'hypothèse de Sallaberry (2004) pour lequel, le cheminement de pensée est intimement lié à la qualité des R1, sans lesquelles la dynamique R1-R2 est alors, de fait, limitée.

À l'issue de cette recherche, nous tentons d'avancer les deux hypothèses suivantes :

- Hypothèse 1: Il est difficile de construire le concept d'élément chimique sans avoir construit son invariant; ce qui pourrait se résumer par "pas de concept sans invariant $\gg$.

- Hypothèse 2: Parvenir à construire son invariant peut faciliter la construction du concept d'élément chimique ; ce qui pourrait se résumer par «tenir l'invariant facilite l'accès au concept $»$. 


\section{Références bibliographiques}

Astolfi, J.-P. et Develay, M. (1989). La Didactique des sciences. Paris : P.U.F.

Bachelard, G. (1938). La formation de l'esprit scientifique. Paris : Vrin.

Bensaude-Vincent, B. (1984). Regards sur l'histoire de l'élément chimique. Bulletin de l'Union des Physiciens, 666, 1273-1284.

Bruner, J. (1996). L'éducation, entrée dans la culture. Paris : Retz.

Bulletin officiel (2005). №5 du Hors-série, 25 Août 2005.

De Crescenzo, L. (1999). Les grands philosophes de la Grèce antique. Paris : De Fallois.

Dupuy, J.-P. (1994). Aux origines des sciences cognitives. Paris : La Découverte.

Fleury, P. (2013) Enseigner le concept d'élément chimique en seconde générale : " quelle stratégie didactique choisir ? », Bulletin de l'union des physiciens, 950, 57-71.

Fleury, P. et Sallaberry J.-C. (2013) Une approche par les concepts pour l'EIST - Le lien invariant-concept comme accès à la transdisciplinarité, Spirale, 52,131-148.

Fleury, P. (2015a) Aborder le concept de pression en seconde générale - démarche et intérêt à faire émerger l'invariant par équilibre qui lui est associé. Bulletin de l'union des physiciens, 972, 367-382.

Fleury, P. (2015b) «Représenter pour qui - Pourquoi représenter» - les représentionsimages et la construction d'un concept scientifique, Année de la recherche en sciences de l'éducation, 131-146. Paris : L'Harmattan.

Laugier, A. et Dumon, A. (2003). Obstacles épistémologiques et didactiques à la construction du concept d'élément chimique : quelles convergences ? Didaskalia, 22, 69-97.

Lefort, M. (2003). Les constituants chimiques de la matière. Paris : Ellipses.

Lemeignan, G. et Weil-Barais, A. (1993). Construire des concepts en physique. Paris: Hachette.

Le Moigne, J.-L. (1995). Les épistémologies constructivistes. Paris : PUF.

Martinand, J.-L. (1986). Connaître et transformer la matière; des objectifs pour l'initiation aux sciences et techniques. Bern : Peter Lang.

Martinand, J.-L. et Viovy, R. (1979). La notion d'élément chimique en classe de cinquième : difficultés, ressources et propositions. Bulletin de l'Union des Physiciens, 613, 878-884.

Piaget, J. (1968). Le structuralisme. Paris : PUF.

Popper, K. (1973). La Logique de la découverte scientifique. Paris : Payot.

Robardet, G. et Guillaud, J.-C. (1997). Eléments de didactique des sciences physiques. Paris : PUF.

Sallaberry, J.-C. (1996). Dynamique des représentations dans la formation. Paris : L'Harmattan.

Sallaberry, J.-C. (2000). Coordination des «représentations image » et des représentations rationnelles dans la construction du concept d'élément chimique. Didaskalia, 17, 101122.

Sallaberry, J.-C. (2004), Dynamique des représentations et construction des concepts scientifiques, Paris : L'Harmattan

Varela, F. (1980). Autonomie et connaissance. Paris : Seuil.

Vergnaud, G. (1985). Concepts et schèmes dans une théorie opératoire de la représentation.

Psychologie Française, 30, 245-252.

Vergnaud, G. (1991). La théorie des champs conceptuels. Recherches en didactique des mathématiques, 10, 133-170

Vergnaud, G. (1995). Apprentissages et Didactiques, où en est-on? Paris : Hachette.

Viovy, R. (1984). La notion d'élément chimique. Bulletin de l'Union des Physiciens, 663, 901-910. 


\section{Annexe 1}

\section{EXPERIENCE 1:}

- Sous la hotte, placer un petit morceau (environ $1 \mathrm{~cm}$ ) de tournure de cuivre dans un tube à essais.

- Verser avec précaution environ $2 \mathrm{~mL}$ d'une solution d'acide nitrique concentré.

- Observer et compléter le document fourni.

\section{EXPERIENCE 2 :}

- Dans un tube à essais, verser environ $1 \mathrm{~mL}$ de la solution obtenue dans l'expérience 1.

- A ce prélèvement, ajouter goutte à goutte une solution concentrée de soude (hydroxyde de sodium).

- A l'aide d'un filtre, séparer les composants du mélange précédent et les récupérer séparément.

- Observer et compléter le document fourni.

\section{EXPERIENCE 3 :}

- Récupérer un peu de solide présent dans le filtre à l'aide d'une spatule et le disposer dans une coupelle en porcelaine. Chauffer au bec bunsen.

- Observer et compléter le document fourni.

\section{EXPERIENCE 4 :}

- Chauffer fortement ( 5 minutes environ) dans un tube, muni d'un tube à dégagement trempant dans de l'eau de chaux, un mélange de poudre de carbone et du solide noir obtenu précédemment.

- Observer et compléter le document fourni. 


\section{Annexe 2 (Feuille A4)}

\begin{tabular}{|c|c|}
\hline Description du document de l'expérience $\mathrm{N}^{\circ} 2$ & Commentaires \\
\hline
\end{tabular}

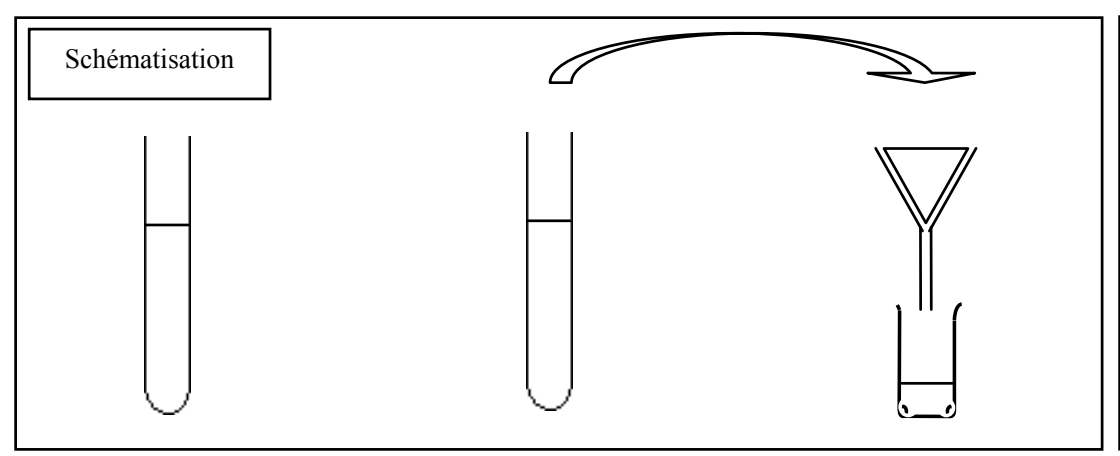

Cette partie est censée permettre de décrire les observables successifs de l'expérience par des R1 sous forme de codages spécifiques (couleurslégendes).

Cette partie est censée permettre de décrire par des représentations $\mathrm{R} 2$ et coordinations R1-R2 les premiers indices favorables à l'émergence de l'invariant. 
Revue « Contextes et Didactiques », nº 6 , décembre 2015

$\underline{\text { Annexe } 3 \text { (Feuille A4) }}$

Mon bilan Que pensez-vous de cette série d'expériences? 
$\underline{\text { Annexe } 4 \text { (Sur la même feuille A4 que l'annexe 1) }}$

\begin{tabular}{|c|c|c|c|c|}
\hline \multicolumn{2}{|c|}{ ESPECES CHIMIQUES } & \multicolumn{2}{|c|}{ CARACTERISTIQUES } & \multirow{2}{*}{ SECURITE } \\
\hline Nom & Symbole & État & Couleur & \\
\hline Métal Cuivre & $\mathrm{Cu}$ & Solide (métal) & orangé & \\
\hline $\begin{array}{l}\text { L'oxyde de } \\
\text { cuivre }\end{array}$ & $\mathrm{CuO}$ & Solide & noir & \\
\hline Carbone végétal & $\mathrm{C}$ & Solide & noir & \\
\hline $\begin{array}{c}\text { L'hydroxyde de } \\
\text { cuivre }\end{array}$ & $\mathrm{Cu}(\mathrm{OH})_{2}$ & Solide & bleu clair & \\
\hline $\begin{array}{c}\text { L'ion } \\
\text { cuivre II }\end{array}$ & $\mathrm{Cu}^{2+}$ & En solution & bleu cyan & \\
\hline $\begin{array}{l}\text { Dioxyde de } \\
\text { carbone }\end{array}$ & $\mathrm{CO}_{2}$ & Gaz & incolore & \\
\hline $\begin{array}{l}\text { Monoxyde } \\
\text { d'azote }\end{array}$ & $\mathrm{NO}$ & Gaz & incolore & \\
\hline Dioxyde d'azote & $\mathrm{NO}_{2}$ & Gaz & roux & \\
\hline Eau & $\mathrm{H}_{2} \mathrm{O}$ & Gaz ou Liquide & incolore & \\
\hline $\begin{array}{c}\text { Solution } \\
\text { d'acide nitrique }\end{array}$ & $\left(\mathrm{H}^{+} ; \mathrm{NO}_{3}^{-}\right)$ & Liquide & incolore & \\
\hline $\begin{array}{l}\text { Solution } \\
\text { de soude }\end{array}$ & $\left(\mathrm{Na}^{+} ; \mathrm{HO}^{-}\right)$ & Liquide & incolore & \\
\hline
\end{tabular}

